\title{
Evaluation of oxidative status in dogs with anemia*)
}

\author{
MEHMET GULTEKIN, HUSEYIN VOYVODA
}

\begin{abstract}
Department of Internal Medicine, Faculty of Veterinary Medicine, Adnan Menderes University, Isikli, Aydin, Turkey
\end{abstract}
\section{Gultekin M., Voyvoda H. \\ Evaluation of oxidative status in dogs with anemia}

Summary

The aim of the present study was to evaluate the relationship between the oxidative status and the severity and type of anemia in dogs. A total of 70 dogs of various breeds, ages and of both sexes were enrolled in the study. Fifty dogs with anemia were classified according to the severity of anemia as mildly $(n=18)$, moderately $(n=18)$ or severely $(n=14)$ anemic on the basis of the hematocrit $(H C T)$ value. Anemia in the same dogs was also classified according to the type as regenerative $(n=26)$ or non-regenerative $(n=24)$ on the basis of the absolute reticulocyte count. Twenty dogs were used as healthy control. Total oxidant status (TOS), total antioxidant capacity (TAC) and malondialdehyde (MDA) values in plasma as well as superoxide dismutase (SOD), glutathione peroxidase (GSH-Px), and catalase (CAT) activities in erythrocyte hemolysate were measured to evaluate the oxidative status. The plasma TOS value was increased in all anemic dogs, irrespective of the severity of anemia, whereas a significant increase in the TAC value and a significant decrease in erythrocyte GSH-Px activity were observed in mild and moderate anemia. Plasma TOS and TAC values were higher in dogs with both types of anemia when compared to the healthy group. A significant increase in plasma MDA concentration and a significant decrease in GSH-Px activity were observed in the non-regenerative anemia group. There were moderate negative correlations between HCT and TOS values in the mild anemia group and between HCT and erythrocyte GSH-Px activity in the regenerative anemia group. In conclusion, oxidative stress develops in dogs with anemia, and it is largely independent of the severity and type of anemia. These results suggest that further studies with different etiologies may also be useful for evaluating the efficacy of antioxidants administered at different doses and in different combinations to treat anemia in dogs.

Keywords: anemia, dog, oxidative stress, biomarker

The basic function of erythrocytes is tissue reoxygenation (22). Erythrocytes are a very important component of antioxidant capacity of blood as well as tissue reoxygenation. They have a strong protective system including non-enzymatic antioxidants, such as glutathione, vitamin $\mathrm{E}$ and ascorbate, as well as important enzymatic antioxidants, such as superoxide dismutase (SOD), glutathione peroxidase (GSH-Px), and catalase (CAT) (3, $16,27)$. On the other hand, erythrocytes are susceptible to oxidative damage due to both oxygen transport and oxidants, such as hydrogen peroxide, superoxide, and hydroxyl, which are present in plasma continuously (11, 24). These oxidants may easily cause lipid peroxidation in erythrocytes, which are very rich in polyunsaturated fatty acids and cholesterol (9). Briefly, erythrocytes are constantly exposed to versatile oxidants and have a complex antioxidant protective system against them (22). Reduced erythrocyte production or increased destruction or loss of erythrocytes that cannot be covered

\footnotetext{
*) This study was partially summarized from the PhD thesis of the corresponding author. The project was funded by Adnan Menderes University Research Projects Funding Unit under project number VTF-12014.
}

by compensation mechanisms results as anemia (10). Anemia may lead to pale mucous membranes, lethargy, exercise intolerance, tachypnea or dyspnea, tachycardia or murmur. Moreover, anemia, depending on its severity and type, may also result in death $(8,28,29)$.

There is a two-way interaction between oxidative damage and anemia. Reduction in the number of erythrocytes results in an impaired antioxidant defense and hypoxia. Hypoxia directly and indirectly contributes to oxidative stress $(10,12,13,24)$. Oxidative stress mostly affects erythrocytes because of the high content of polyunsaturated fatty acids in their membrane. Erythrocytes, which have a hardened membrane structure and cannot change their shape, are phagocytized by the spleen and macrophages (24). Thus, oxidative damage shortens the life span of erythrocytes and causes or exacerbates anemia $(10,17)$. Anemia can occur in many disease processes or as a result of diseases, and it is a common sign in dogs (8). There is no completely effective antianemic drug for all types of anemia. Therefore, treatment consists mostly in symptomatic or supportive practices as well as in removal of etiology (15). The type and 
severity of anemia should be known in order to assess its etiology, the prognosis, and the primary intervention in treatment (21).

The oxidative status has been evaluated in different types of anemia in humans $(2,13,24)$. However, the relationship between the oxidative status and the severity and type of anemia in dogs has been reported only in renal anemia (14). Determination of the oxidative status in dogs with anemia of different types and severity may also be useful in assessing the advisability of antioxidant application. Therefore, this study aimed to evaluate the oxidative status and its relation with the type and severity of anemia in dogs.

\section{Material and methods}

Animal population and sample collection. This research was approved by the Adnan Menderes University Animal Experiments Local Ethics Committee (permit number B.30.2. ADÜ.0.00.00.00/050.04/2011/075). Informed written consent was obtained from all owners prior to enrolment.

Dogs were selected from animals presented for examination or treatment between March 2013 and October 2013 to the Small Animal Clinic of the Department of Internal Medicine of the Adnan Menderes University, Faculty of Veterinary Medicine. Following clinical examination of the dogs, laboratory analyzes were conducted. A total of $70 \mathrm{dogs}$ of various breeds, ages and sexes were enrolled in the study. Fifty dogs with anemia included 22 females and 28 males, and their average age was $5.2 \pm 2.3$ years (ranging from 1 to 11 years). Anemia was classified as mild $(\mathrm{n}=18)$, moderate $(n=18)$ or severe $(n=14)$ on the basis of the HCT value, and as regenerative $(n=26)$ or non-regenerative $(n=24)$ on the basis of the absolute reticulocyte count. Twenty dogs, shown to be healthy by clinical and laboratory examinations, were used as control. The group of healthy dogs consisted of 8 females and 12 males, and their average age was $4.6 \pm 2.2$ years (ranging from 1 to 9 years).

Blood samples were taken once from vena cephalica antebrachi into heparinized ethylenediaminetetraacetic acid (EDTA) tubes and non-anticoagulant tubes. Complete blood count and absolute reticulocyte count were performed shortly after sample collection. Plasma was obtained by centrifugation of heparinized blood, and erythrocyte hemolysate was prepared from the rest of the sample. Samples were stored at $-20^{\circ} \mathrm{C}$ for analysis of total antioxidant capacity (TAC), total oxidant status (TOS) and malondialdehyde (MDA) concentrations in plasma and activity of SOD, CAT, GSH-Px enzymes in erythrocyte hemolysate.

Hematological and biochemical analysis. Complete blood counts were determined with an automatic blood counting device (Abacus Junior Vet 5, Diatron, Hungary). in dogs $(\mathbf{p}<0.05)$
Anemic dogs were classified as mildly (\% 30-36), moderately $(\% 20-30)$, severely $(\% 20-13)$ or very severely $(\%<13)$ anemic on the basis of the hematocrit (HCT) value (29).

Absolute reticulocyte count was performed manually by the New Methylene Blue staining method (5). For this purpose, EDTA whole blood was mixed with the same amount of New Methylene Blue and kept at room temperature for 15 minutes. The mixture was dropped on a slide, and erythrocytes containing RNA particles were counted at $\times 1000$ magnification (CX31, Olympus, Japan). The absolute reticulocyte count was obtained by evaluating at least 1000 erythrocytes and multiplying the number of erythrocytes by the percentage of reticulocytes. Anemia was classified as non-regenerative $(<80.000)$ or regenerative $(<80.000)$ on the basis of the absolute reticulocyte count (5).

TAC and TOS levels were measured with commercially available test kits by the methods described by Erel $(6,7)$ (Rel Assay Diagnostics, Turkey). Erythrocyte SOD and GSH-Px activities were measured with commercial test kits (Randox, United Kingdom). An automated clinical chemistry analyzer (Sinnowa D280, China) was used for the estimation of TAC, TOS, SOD and GSH-Px levels. MDA concentration was measured by the method described by Yoshoiko et al. (31), and erythrocyte CAT activity was measured by the method described by Luck et al. (19) with a spectrophotometer (Shimadzu, Japan).

Statistical analysis. Statistical analysis was carried out with statistical software (SPSS 22.0, SPSS Inc., Chicago, USA). One-way analysis of variance was used for the comparison, and Tukey's test was used for post hoc analysis to determine differences in the biochemical parameters of the groups. A Pearson or Spearman test was used to evaluate the correlation between the type and severity of anemia and the parameters associated with oxidative status. The correlation coefficient was evaluated as high $(\mathrm{r}>60)$, moderate $(r=30-60)$ or poor $(r<30)$. Probability values of $p<0.05$ were considered significant.

\section{Results and discussion}

Differences in TOS and TAC values and GSH-Px activity between the groups classified according to the severity of anemia were statistically significant (Tab. 1). In this context, plasma TOS values were found to be higher in the mild, moderate and severe

Tab. 1. HCT value, reticulocyte count and oxidative status according to the severity of anemia

\begin{tabular}{|l|c|c|c|c|c|}
\hline \multicolumn{1}{|c|}{ Parameters } & \multirow{2}{*}{ Healthy $(\mathrm{n}=20)$} & \multicolumn{3}{c|}{ Severity of anemia } & $\mathrm{p}=$ \\
& & Mild $(\mathrm{n}=18)$ & Moderate $(\mathrm{n}=18)$ & Severe $(\mathrm{n}=14)$ & \\
\hline HCT $(\%)$ & $43.32 \pm 4.90$ & $33.25 \pm 1.60$ & $26.17 \pm 2.57$ & $17.87 \pm 1.53$ & 0.000 \\
Reticulocyte $(/ \mu \mathrm{L})$ & $47341 \pm 2164^{\mathrm{a}}$ & $111796 \pm 5356^{\mathrm{b}}$ & $90261 \pm 5528^{\mathrm{a}}$ & $58982 \pm 4165^{\mathrm{a}}$ & 0.001 \\
\hline TOS $\left(\mu \mathrm{mol} \mathrm{H} \mathrm{O}_{2}\right.$ Equiv./L) & $4.27 \pm 0.86^{\mathrm{a}}$ & $7.05 \pm 2.51^{\mathrm{b}}$ & $6.87 \pm 1.97^{\mathrm{b}}$ & $6.69 \pm 1.04^{\mathrm{b}}$ & 0.000 \\
TAC $(\mathrm{mmol}$ Trolox Equiv./L) & $0.74 \pm 0.08^{\mathrm{a}}$ & $0.88 \pm 0.13^{\mathrm{b}}$ & $0.92 \pm 0.13^{\mathrm{b}}$ & $0.82 \pm 0.15^{\mathrm{a}}$ & 0.000 \\
\hline MDA $(\mu \mathrm{mol} / \mathrm{L})$ & $16.04 \pm 5.24$ & $19.88 \pm 7.07$ & $22.01 \pm 13.15$ & $20.61 \pm 6.98$ & 0.077 \\
SOD $(\mathrm{U} / \mathrm{g} \mathrm{HGB})$ & $816.77 \pm 87.19$ & $853.22 \pm 201.32$ & $945.05 \pm 212.40$ & $890.85 \pm 184.08$ & 0.154 \\
\hline GSH-Px $(\mathrm{U} / \mathrm{g} \mathrm{HGB})$ & $472.43 \pm 7^{\mathrm{a}}$ & $400.47 \pm 123.8^{\mathrm{b}}$ & $405.80 \pm 99.0^{\mathrm{b}}$ & $470.68 \pm 88.4^{\mathrm{a}}$ & 0.021 \\
\hline CAT $(\mathrm{U} / \mathrm{g} \mathrm{HGB})$ & $6.07 \pm 2.20$ & $5.44 \pm 2.46$ & $5.06 \pm 2.09$ & $4.60 \pm 2.39$ & 0.294 \\
\hline
\end{tabular}

Explanations: $\mathbf{a}, \mathbf{b}$ - different letters indicate significant differences between rows and columns 
anemia groups compared to the healthy group $(\mathrm{p}<0.001)$, and the differences between the anemia groups were not statistically significant. Increased plasma TAC values were found in the mild and moderate anemia groups compared to the healthy group $(\mathrm{p}<0.001)$. Erythrocyte GSH-Px activity was found to be lower in the mild and moderate anemia groups compared to the healthy and severe anemia groups $(\mathrm{p}<0.05)$.

Differences in TOS and TAC values, MDA concentration and GSH-Px activity between the groups classified according to the type of anemia were statistically significant (Tab. 2).

Plasma TOS and TAC values were higher in the regenerative and non-regenerative anemia groups than they were in the healthy group $(p<0.001)$. The plasma MDA concentration was higher in the non-regenerative group compared to the healthy $(\mathrm{p}<0.001)$ and regenerative anemia groups $(\mathrm{p}<0.05)$. Erythrocyte GSH-Px activity was lower in the non-regenerative anemia group $(\mathrm{p}<0.05)$ compared to the healthy group. Erythrocyte SOD and CAT activities did not show statistically significant differences between the groups.

There was a moderate negative correlation between HCT and TOS values $(p<0.05)$ in the mild anemia group, while moderate negative correlations were found between HCT and erythrocyte GSH-Px activity $(p<0.01)$ in the regenerative anemia group. However, the relationships between the other variables and the HCT value and reticulocyte count in the groups were not statistically significant.

In relation to the oxidative status of the organism, TAC and TOS are evaluated as total biomarkers of the oxidant/antioxidant balance. SOD, GSH-Px and CAT enzymes are evaluated as individual biomarkers of enzymatic antioxidants. MDA is a lipid peroxidation product used as a marker of oxidative damage to lipids. Increases in TOS and MDA concentrations and decreases in TAC, SOD, GSH-Px and CAT levels are mainly regarded as indicative of oxidative stress $(3,6,7,9,27)$. In the present study, the effects of the severity and type of anemia on the oxidative status were evaluated in dogs.

In humans, the oxidative status has been investigated in anemia of different types and severity $(2,12,13,20$, 24). However, the oxidative status in dogs with anemia has been investigated mostly on the basis of infections that result in anemia. In infectious diseases, the activation of macrophages and production of reactive oxygen species (ROS) for phagocytizing the agent are shown as important causes of increased oxidative stress (18). In addition, it has been found that, in dogs with babesiosis, reactive species produced by the agent as well as the direct breakdown of erythrocytes also contribute to oxidative stress and anemia $(4,23)$. Oxidative stress in dogs with leishmaniasis has also been associated with chronic renal failure and uremia caused by the disease (1). Only one study, in dogs with anemia due to chronic renal failure, evaluated the relationship between the oxidative status and the severity of anemia (14). In the study, there was a negative correlation between the HCT value and the TBARS level in anemic dogs due to chronic renal failure in both groups (HCT $>22 \%$, HCT $\leq 22 \%$ ), and the development of anemia was associated with uremic toxins and the chronic inflammatory process. However, it was also emphasized that there was no strong correlation between oxidative stress and the severity of anemia (14).

In the present study, the plasma TOS value was increased in the mild, moderate and severe anemia groups, and the plasma TAC value was increased in the mild and moderate anemia groups compared to the healthy group. An increased concentration of TOS indicates that the oxidant/antioxidant balance is impaired in dogs with anemia of different severity. The increased value of TAC may be due to a compensatory response in mild to moderate anemia (4). It has been reported that the organism exposed to mild or moderate oxidative stress activates antioxidant systems more efficiently in the initial period $(14,27)$. For this reason, in the first stage, TAC and the levels of individual antioxidants in different structures may not be reduced, or may even be increased (4).

Erythrocytes are rich in polyunsaturated fatty acids and cholesterol, and can therefore be easily exposed to lipid peroxidation (9). Increased plasma MDA concentration and decreased GSH-Px activity were significant in the non-regenerative anemia group, whereas plasma TAC and TOS values were higher in dogs with both types of anemia. These changes in the oxidative status in both types of anemia may be associated with oxidative stress. The higher plasma MDA concentration and the lower erythrocyte GSH-Px activity in the non-regenerative anemia group can be attributed to the fact that developing oxidative stress is more severe in non-regenerative anemic dogs compared to the regenerative anemia group. The severity of anemia may be reduced by increasing erythropoiesis in regenerative anemia $(8,10)$. However, 
due to restricted or blocked erythropoiesis, the deficit of erythrocytes becomes more severe in non-regenerative anemia, which leads to more intensive exposure to ROS $(16,24)$. Additionally, it has been found that SOD and CAT activities are higher in reticulocytes $(26,30)$. In the current study, SOD and CAT activities were not significantly different in the regenerative and non-regenerative anemia groups compared to healthy dogs. However, the increase in TAC levels in both types of anemia could be interpreted as a response of the organism exposed to oxidative stress at various levels, which activates nonenzymatic antioxidant systems $(4,27)$.

In the current study, the activity of GSH-Px was found to be significantly lower in the non-regenerative, mild and moderate anemia groups compared to the healthy dogs. The main function of GSH-Px is to reduce lipid peroxides to non-toxic alcohols (27). GSH-Px reacts with oxidants such as hydrogen peroxide and lipid hydroperoxides produced in the membrane $(16,22)$. It is believed that hydrogen peroxide, which cannot be neutralized, is increased on the membrane surface as a result of decreased GSH-Px activity $(24,25)$. Besides, it was determined that MDA concentration, which is a marker of lipid peroxidation, was significantly higher in the dogs with non-regenerative anemia. Therefore, decreased levels of GSH-Px activity may be attributed to the use of this enzyme to neutralize lipid peroxidation products.

In the present study, the relationships between oxidative status biomarkers and the type and severity of anemia were found to be limited. There was a moderate negative correlation between HCT and TOS values in the mild anemia group, and between HCT and erythrocyte GSH-Px activity in the regenerative anemia group. This may be related to the lack of etiologic differentiation of dogs with anemia. Besides, the absence of a meaningful correlation may also be related to the limited number of anemic dogs.

In conclusion, according to the results of the present study, oxidative stress develops in dogs with anemia. However, it is largely independent of the severity and type of anemia. The serum TOS, TAC and MDA values and GSH in erythrocytes appear to be sensitive indicators of oxidative stress in the course of anemia and may be recommended as good markers in small animal practice. These results suggest that further studies with different etiologies may also be useful for evaluating the efficacy of antioxidants administered at different doses and in different combinations to treat anemia in dogs.

\section{References}

1. Almeida B. F. M., Narciso L. G., Melo L. M., Preve P. P., Bosco A. M., Lima V. M. F., Ciarlini P. C.: Leishmaniasis causes oxidative stress and alteration of oxidative metabolism and viability of neutrophils in dogs. Vet. J. 2013, 198, 599-605

2. Aslan M., Horoz M., Celik H.: Evaluation of oxidative status in iron deficiency anemia through total antioxidant capacity measured using an automated method. Turk. J. Hematol. 2011, 28, 42-46.

3. Chapple I. L.: Reactive oxygen species and antioxidants in inflammatory diseases. J. Clin. Periodontol. 1997, 24, 287-296.
4. Chaudhuri S., Varshney J. P., Patra R. C.: Erythrocytic antioxidant defense, lipid peroxides level and blood iron, zinc and copper concentrations in dogs naturally infected with Babesia gibsoni. Res. Vet. Sci. 2008, 85, 120-124.

5. Cowgill E. S., Neel J. A., Grindem C. B.: Clinical application of reticulocyte counts in dogs and cats. Vet. Clin. North. Am. Small. Anim. Pract. 2003, 33, 1223-1244.

6. Erel $O$.: A new automated colorimetric method for measuring total oxidant status. Clin. Biochem. 2005, 38, 1103-1111.

7. Erel O.: A novel automated direct measurement method for total antioxidant capacity using a new generation, more stable ABTS radical cation. Clin. Biochem. 2004, 37, 277-285.

8. Grimes C. N., Fry M. M.: Nonregenerative anemia: mechanisms of decreased or ineffective erythropoiesis. Vet. Pathol. 2015, 52, 298-311.

9. Grotto D., Santa Maria L., Valentini J., Paniz C., Schmitt G., Garcia S. C. Importance of the lipid peroxidation biomarkers and methodological aspects for malondialdehyde quantification. Quim. Nova 2009, 32, 169-174.

10. Harvey W. J.: Erythrocyte biochemistry, [in:] Weiss D. J., Wardrop K. J.: Schalm's Veterinary Hematology. $6^{\text {th }}$ Edition. Blackwell Publishing, Iowa, USA 2010, p. 131-135

11. Hess J. R.: Red cell storage. J. Proteomics. 2010, 73, 368-373.

12. Iuchi Y.: Anemia caused by oxidative stress, [in:] Silverberg D.: Anemia. InTech, Croatia 2012, p. 49-62.

13. Khanna A., Dubey M., Sinha R.: Oxidative stress and its correlation with severity of maternal anemia. Indian. J. Prev. Soc. Med. 2010, 41, 231-236.

14. Kogika M. M., Lustoza M. D., Hagiwara M. K., Caragelasco D. S., Martorelli C. R., Mori C. S.: Evaluation of oxidative stress in the anemia of dogs with chronic kidney disease. Vet. Clin. Path. 2014, 44, 70-78.

15. Lanevschi A., Wardrop K. J.: Principles of transfusion medicine in small animals. Can. Vet. J. 2001, 42, 447-454.

16. Lang F., Abed M., Lang E., Föller M.: Oxidative stress and suicidal erythrocyte death. Antioxid. Redox. Signal. 2014, 21, 138-153.

17. Lang F., Lang K. S., Lang P. A., Huber S. M., Wieder T.: Mechanisms and significance of eryptosis. Antioxid. Redox. Signal. 2006, 8, 1183-1192.

18. Longoni S. S., Sánchez-Moreno M., López J. E. R., Marín C.: Leishmania infantum secreted iron superoxide dismutase purification and its application to the diagnosis of canine Leishmaniasis. Comp. Immunol. Microbiol. Infect. Dis. $2013,36,499-506$

19. Luck H.: Catalase, [in:] Bergmeyer H. U.: Methods of enzymatic assays Academic Press, New York, USA 1965, p. 885-894.

20. Madhikarmi N. L., Murthy K. R. S.: Antioxidant enzymes and oxidative stress in the erythrocytes of iron deficiency anemic patients supplemented with vitamins. Iran Biomed. J. 2014, 18, 82-87.

21. Mills J.: Anaemia, [in:] Day M. J., Kohn B.: BSAVA Manual of Canine and Feline Haemotology and Transfusion Medicine, $2^{\text {nd }}$ Edition. BSAVA, England 2012, p. 31-44

22. Mohanty J. G.: Red blood cell (RBC) oxidative stress contributes to reduced RBC deformability and oxygen delivery leading to the occurrence of anemia J. Blood Disord. Transfus. 2013, 4, 58-66.

23. Murase T., Ueda T., Yamato O., Tajima M., Maede Y.: Oxidative damage and enhanced erythrophagocytosis in canine erythrocytes infected with Babesia gibsoni. J. Vet. Med. Sci. 1996, 58, 259-261

24. Nagababu E., Gulyani S., Earley C. J., Cutler R. G., Mattson M. P., Rifkind J. M. Iron-deficiency anaemia enhances red blood cell oxidative stress. Free Radic. Res. 2008, 42, 824-829.

25. Niedzwiedz A., Jaworski Z.: Oxidant-antioxidant status in the blood of horses with symptomatic Recurrent Airway Obstruction (RAO). J. Vet. Intern. Med. 2014, 28, 1845-1852.

26. Otsuka Y., Yamasaki M., Yamato O., Maede Y.: The effect of macrophages on the erythrocyte oxidative damage and the pathogenesis of anemia in Babesia gibsoni-infected dogs with low parasitemia. J. Vet. Med. Sci. 2002, 64, 221-226.

27. Tanja P., Alenka N. S., Butinar J., Nataša T., Marija P., Bettina K., Kessler M. Antioxidant status in canine cancer patients. Acta Vet. (Beogr). 2008, 58, 275$-286$.

28. Thrall M. A.: Classification of and diagnostic approach to anemia, [in:] Thrall M. A., Weiser G., Allison R., Campbell T. W.: Veterinary Hematology and Clinical Chemistry, $2^{\text {nd }}$ Edition. Wiley-Blackwell, USA 2012, p. 75-113.

29. Tvedten H.: Laboratory and clinical diagnosis of anemia, [in:] Weiss D. J., Wardrop K. J.: Schalm's Veterinary Hematology, $6^{\text {th }}$ Edition. Blackwell Publishing, Iowa, USA 2010, s. 152-161.

30. Yamasaki M., Otsuka Y., Yamato O., Tajima M., Maede Y.: The cause of the predilection of Babesia gibsoni for reticulocytes. J. Vet. Med. Sci. 2000, 62, 737-741.

31. Yoshoiko T., Kawada K., Shimada T.: Lipid peroxidation in maternal and cord blood and protective mechanism against active oxygen toxicity in the blood. Am. J. Obstet. Gynecol. 1979, 135, 372-376.

Corresponding author: Mehmet Gultekin, DVM, PhD; Department of Internal Medicine, Faculty of Veterinary Medicine, Adnan Menderes University, Isikli, Aydın, 09017, Turkey; e-mail: gultekinmehmet@gmail.com 